\title{
La teoría propensivista como base metafísica para la construcción de una cosmología en el pensamiento de Karl Popper
}

\author{
David Mayo Sánchez \\ Universidad de Salamanca \\ damayo1@usal.es
}

\section{Introducción}

El marcado interés cosmológico de la filosofía de Karl Popper, ya manifestado desde sus primeras obras de carácter estrictamente epistemológico ${ }^{1}$, fue dando lugar a un posterior programa especulativo que diera cuenta de las grandes preguntas que el pensamiento filosófico había planteado desde sus orígenes, a saber, cuál es la naturaleza o estructura del mundo o cómo se podría construir una imagen unificada de la realidad, que a la vez explique la gran diversidad de elementos que la componen. Para Popper, esta labor no recae solamente sobre el conocimiento que mejor explica la realidad, es decir, la ciencia, sino que ésta requiere de la filosofía -en este caso de la metafísica-, para que juntamente con

1 Ya en el prefacio de la Lógica de la investigación científica Popper afirma: "existe un problema filosófico por el que se interesan todos los hombres que reflexionan: es el de la cosmología, el problema de entender el mundo-incluidos nosotros y nuestro conocimiento como parte de él-. Creo que toda la ciencia es cosmología, y, en mi caso, el único interés de la filosofía, no menos que el de la ciencia, reside en las aportaciones que ha hecho a aquélla", en (2013; 21). 
ella se logre esa visión de conjunto y, a su vez, se puedan regular y precisar las mismas teorías científicas; esto es, que para Popper una metafísica es posible solamente en relación con las ciencias naturales, y es esa metafísica la que el filósofo vienés se propone construir. En palabras de Popper: "una nueva metafísica de la física, un nuevo programa de investigación para la física, que unifique la mayoría de sus programas más antiguos y que, además, parece ofrecer posibilidades para una unificación de las ciencias física y biológica" (2011a: 400).

Es así cómo a partir de la teoría científica fundamental de la realidad física, tal es la teoría cuántica, que Popper asienta su teoría de las propensiones, que es con la que hace una reinterpretación del cálculo de probabilidades, al mismo tiempo que una superación de las interpretaciones subjetivista y frecuencial de la probabilidad -las cuales son el soporte formalista generalmente aceptado para explicar el indeterminismo presente en dicha teoría-, ya que estas interpretaciones, sostiene Popper, dan lugar a "ciertos elementos perturbadores de un carácter irracional y subjetivista" (2011a: 391). Esto es así porque dichas interpretaciones de la probabilidad propician, principalmente, una epistemología subjetivista "causada por una limitación fundamental e irreducible de nuestro conocimiento" (2011c: 28-29), lo que para Popper es inadmisible, debido a su radical inclinación a la descripción objetiva del mundo y a su decidido realismo metafísico, que son los presupuestos necesarios para que la ciencia pueda describir y explicar adecuadamente la realidad, y así evitar lo que no es ciencia, sino dudosa filosofía de corte idealista, fenomenalista o incluso positivista.

Es, especialmente, desde la superación de la teoría estadístico frecuencial de la probabilidad aplicada a la teoría 
cuántica -interpretación dependiente de mediciones y, que por ende, no da razón de la realidad en sí, de la estructura propia del mundo físico-, que Popper busca señalar las implicaciones metafísicas que esta situación comporta. Esto lleva al filósofo a postular el propensivismo como la solución que logre fundamentar metafísicamente un indeterminismo exigido de una regularidad estructural ontológica o, en términos popperianos: "propiedades relacionales de la situación objetiva total” (2011a: 398). Además, superar el formalismo matemático que simplemente ve en las teorías científicas un instrumento para interpretar o predecir los resultados de los experimentos, es decir, meras aplicaciones práctico-formalistas -y no un medio para entender y explicar el mundo-, como es el caso de la interpretación instrumentalista del dualismo onda-partícula (principio de complementariedad), el cual no resulta equivalente físicamente o posible como una situación física real, y que igualmente ha generado ambigüedad conceptual y disparidad teórica en la ciencia. Es aquí nuevamente donde la interpretación propensivista popperiana busca ofrecer la unificación de los criterios científicos, suplantar metafísicamente interpretaciones insuficientes $y$, con ello, afirma el filósofo, obtener un sentido filosófico coherente de la teoría cuántica y, por tanto, "proporcionar una perspectiva coherente del mundo físico" (2011c: 211).

Toda esta especulación filosófica tiene como fin trascender las consecuencias puramente científicas, para poder así establecer una concepción de mundo como un universo abierto, emergente y en devenir, que juntamente explique sus diferentes niveles de realidad con su respectiva interacción. Es aquí donde Popper sitúa su propuesta de los Tres Mundos, como consecuencia de la emergencia-creatividad proveniente de un universo abierto, afirmando de esta manera la plura- 
lidad presente en el universo, al igual que la diferenciación y la interdependencia mutua entre dichos niveles de realidad; lo que da como resultado -a un nivel general- una estructura indeterminista y causal de lo real.

Para ello, la ontología de la ciencia popperiana se cimienta sobre un realismo metafísico, el cual se constituye como el sustrato para una concepción general y consistente de la estructura del mundo para con ello eliminar el instrumentalismo científico que se cierne como una especie de sofisticación intelectual que pretende reducir el conocimiento "científico" a puro formalismo, a utilidad y a subjetivismo, lo que para Popper representa una visión tergiversada del saber científico, además de una errada concepción filosófica del mundo y del hombre. Es por todo ello que el filósofo austríaco propone un fundamento para toda la edificación científica y metafísica que conforma su posterior cosmología; esto, creemos que lo realiza apelando a un soporte imprescindible de tipo ontológico, como lo es la causalidad, la cual, asimismo, identificamos y apoyamos en la teoría metafísica de las propensiones.

El realismo metafísico

“La argumentación critica con el propósito de acercarse más a la verdad, carecería de sentido sin una realidad objetiva, un mundo cuyo descubrimiento hacemos nuestra tarea" (2011a: 121). Con estas palabras, el filósofo vienés señala claramente su insigne proyecto intelectual, éste es llegar a la realidad tal como ella es, con el fin de hacerla inteligible a la mente, a la total existencia humana y, con ello, oponerse a todo intento y pretensión irracionalista que impida tal labor. Es así como Popper anuncia un racionalismo promotor de la objetividad 
del mundo físico, de un sistema legaliforme ${ }^{2}$ perteneciente a una realidad que como tal es independiente de la mente, que es base para poder construir teorías acertadas, las cuales "son genuinas conjeturas, suposiciones acerca del mundo, de alto contenido informativo" $(1972 ; 150)$ y, que a su vez, posibilitan una descripción real del mundo. Pero cuando las teorías resultan erradas es porque la previa especulación teórica que las ha propiciado no ha hecho caso de la racionalidad crítica que tiene como fin eliminar los errores ontológicos y epistemológicos que le dan la preeminencia a las experiencias subjetivas, a la mediación determinante del sujeto cognoscente antes que a la realidad en sí.

Es esa intrusión del subjetivismo en el conocimiento, y por tanto en la ciencia, la que Popper busca rebatir, puesto que en palabras del filósofo: "nos dice que construyamos el mundo físico a partir de mi propia «egocéntrica» experiencia perceptual" (2011a: 143), y dicha construcción de tipo idealista se topa con ciertos inconvenientes -recibe una patada- del realismo de sentido común, que impiden al subjetivismo avanzar hacia elaboraciones que van en contra de la estructura de la realidad, ya previamente dada y no construida por una con-

2 Popper sostiene que las cosas son lo que son porque son configuradas o constituidas por algo otro, en este caso las leyes de la naturaleza, que son las que disponen necesariamente las propiedades o componentes que forman parte de cada cosa o hecho particular en sí. Con esto, las leyes o teorías adquieren un carácter explicativo-necesario debido a la inexistencia de una experiencia pura y a la necesidad de explicar estas experiencias, lo cual requiere de teorías explicativas que por su parte trascienden la experiencia pura (y por ello no son verificables empíricamente). Esto significa que todas las cosas poseen constitutivamente un comportamiento legal. Así, afirma: "Las leyes (que, a su vez, necesitan explicación ulterior) explican, pues, las regularidades o similaridades entre cosas individuales o hechos o sucesos singulares. [...] Las leyes de la naturaleza se conciben, por el contrario, como descripciones (conjeturables) de las propiedades estructurales ocultas en la naturaleza, de nuestro propio mundo" (en Popper, 2011a: 177). 
ciencia subjetiva. "Cuando [el investigador] fracasa no le echa la culpa al mundo sino a sus limitaciones personales o la falta de medios. Cuando triunfa se siente satisfecho porque sus ideas se conforman en alguna medida a la realidad, o la transforman eficazmente según diseños y planes fundados sobre un trozo de conocimientos de cosas reales"' (Bunge, 1985: 53). Ante esto, Popper igualmente considera que el conocimiento objetivo -entendido como científico- se da a partir de unos contenidos cognoscitivos extraídos de la realidad extramental y, que simultáneamente están necesitados de su correspondiente asimilación teórica, realizada, claro está, por un sujeto cognoscente ya obligado a ajustarse a los previos contenidos teórico-objetivos ${ }^{3}$. Así que no es posible un conocimiento fundado en una certeza subjetiva de tipo cartesiana, a saber, en datos radicados en experiencias empíricas, ya que esos datos ya son una primigenia interpretación específica que remite a un conocimiento previamente construido desde una matriz teórica ya constituida y aceptada de manera racional debido a que resultó ser la más informativa. Así, escribe Popper:

Los subjetivistas suponen acríticamente que sobre la base de esos «datos» puede erigirse el edificio del conocimiento -del conocimiento científico-. [...] Pero no existen: no existen los «datos» sin interpretar; nada que tomar como base. Todo nuestro conocimiento es interpretación a la luz de nuestras expectativas,

3 En su teoría de los Tres Mundos, Popper afirma que en el conocimiento objetivo del Mundo 3 existen objetos autónomos (no procedentes de los Mundos 1 y 2 , pero siempre vinculados o remitidos a los mismos) respecto a nuestros procesos de pensamiento, y que de hecho influyen decididamente en nuestros procesos de pensamiento. Véase, por ejemplo, Popper, 2010: 143-148. 
nuestras teorías, y es, por tanto, hipotético de una u otra manera ${ }^{4}$ (2011a: 142).

Esas teorías científicas, construidas desde hipótesis deductivas $\mathrm{y}$, al mismo tiempo, testadas contra los hechos, se pueden clasificar como mejores o peores, esto a partir de su pervivencia a los tests cruciales realizados, por supuesto, desde la base observacional. Así, las teorías que ofrezcan un alto contenido informativo, que resuelvan más problemas de los presentados por la realidad y ofrezcan las explicaciones satisfactorias, contribuyen al desarrollo del conocimiento científico, nunca completo, pleno o acabado, sostiene firmemente Popper, puesto que con el aporte teórico exitoso -si realmente es exitoso- vienen nuevos problemas de mayor profundidad, esto debido a que Popper considera contundentemente que la realidad es inagotable, es decir, que ninguna teoría por más satisfactoria que sea puede explicar plenamente la realidad. Es por ello que el filósofo sostiene categóricamente que no hay certeza absoluta en la ciencia.

Pero a pesar de la falibilidad del conocimiento científico (y del conocimiento en general), es posible y, más aún, deseable, que tal conocimiento haga referencia a la realidad extramental, que sea seguro, objetivo y fundamentalmente verdadero. Esto significa que las teorías científicas tienen que ser verdaderas, y son verdaderas cuando hacen referencia y responden a los hechos ${ }^{5}$; no obstante, esa ver-

4 Popper es defensor de lo que se conoce como la Carga teórica de los hechos (además de otros pensadores como P. Duhem, O. Neurath.), la cual sostiene que las observaciones presuponen una conceptualización teórica; esto en contra de la tesis sostenida desde la Concepción Heredada, que apoya la supuesta neutralidad de las observaciones empíricas. Véase, por ejemplo, Popper, 2011a: 171-189.

5 Popper sostiene que el objetivo de la ciencia es encontrar explicaciones satisfactorias, esto bajo la forma universalmente aceptada para la explicación 
dad que es exigida para las teorías y que es colocada como el criterio de garantía para diferenciar las teorías verdaderas de las falsas o de la simple creencia, nunca es poseída de manera total, sino simplemente lo que se puede lograr son acercamientos a ella; ésta es la idea de verosimilitud que postula Popper, en cuanto a grados de correspondencia para con la verdad. Aquí es notorio que el concepto popperiano de verdad nuevamente hace una remisión obligatoria a la realidad -reafirmando así su realismo metafísico-, a su limitado acceso y, con ello, esa pretendida obtención de la verdad también adquiere el mismo carácter parcial, para convertirse en un elemento regulador de las teorías en su acceso a la realidad en sí. Esto significa que la idea de verdad objetiva cumple una función delimitadora, de ajuste o incluso de canon, para poder así identificar los errores que surgen del quehacer indagatorio de la ciencia cuando no corresponde a ningún hecho (a la realidad) y, de esta manera, señalar las teorías falsas. "Así, por medio de la noción de 'verdad', Popper configura su realismo científico, es decir, la tesis según la cual existe un mundo externo que es cognoscible por la Ciencia" (Martínez, 2005: 188). Popper lo expone de la siguiente manera:

Si no somos realistas, difícilmente podremos comprender cuál es la tarea de la ciencia que, como he apuntado, consiste en dar con explicaciones satisfactorias [...] que difícilmente se puede comprender sin recurrir a la de descubrimiento, a la de progreso ha-

científica: explicandum (acontecimiento concreto), aquello por explicar y explicans (leyes generales) lo que explica, (en 2013: 72ss; también en Hempel, 2005: 325-332). Además, según Popper, el explicandum debe ser verdadero, es decir, debe hacer referencia a hechos verdaderos y no imaginarios (ver Popper, 2011a: 171-189). 
cia estratos más profundos de explicación: son la idea de que hay algo que descubrir y discutir críticamente (2011a: 245).

Crítica al instrumentalismo probabilista

A partir de estos antecedentes, la crítica de Popper se dirige contra lo que se opone a su realismo - el cual está en pro de la aproximación a la verdad, al conocimiento objetivo de la realidad-, a saber, la concepción instrumentalista de las teorías científicas. El instrumentalismo, según Popper, tiene sus principales planteamientos en la crítica que hace Berkeley a la nueva ciencia que incursionaba de la mano de Newton, describiéndola como una mera "hipótesis matemática", para el "cálculo y la predicción de los fenómenos o apariencias" (1972: 132), y que ha llegado a convertirse para la teoría física actual en un dogma aceptado y considerada como la concepción oficial. Esta instauración dominante del instrumentalismo en la ciencia se ha logrado, según el filósofo, por un problema de comprensión -radicado indudablemente en el rechazo al realismo metafísico y sus derivaciones- aplicado a las teorías científicas, específicamente a la teoría cuántica y el correspondiente éxito práctico de sus aplicaciones puramente formalistas. Aquí, la teoría cuántica, de la cual parte Popper para postular su ontología de la ciencia, representa un cambio paradigmático en las concepciones filosóficas de la realidad y en cuanto a la posición del indagador. Esta teoría científica trata y altera algunos conceptos propiamente filosóficos que son fundamentales para la disposición e inteligibilidad de lo real, como lo son el determinismo, la causalidad, el objetivismo y el realismo, principalmente, y que son a los que Popper busca aplicar su crítica filosófica. 
Popper acepta la teoría cuántica como teoría fundamental de la física, pero lo que no acepta es la concepción contraria a sus tesis realistas, es decir, la llamada interpretación de Copenhague -el dogma dominante-, que describe, siguiendo la declaración de Werner Heisenberg y las tesis de Niels Bohr, con estas palabras: " «la realidad objetiva se ha esfumado» y que la mecánica cuántica no presenta partículas, sino más bien nuestro conocimiento, nuestra observación, nuestra conciencia de las partículas"'6 (2011c: 57). De esta manera, la tesis sostenida por la escuela de Copenhague parece sugerir, a partir del probabilismo procedente del indeterminismo cuántico, que la conciencia del investigador (observador) cumple una función determinante en la constitución misma de la realidad, en este caso la realidad microscópica, la estructura fundamental del mundo físico y, con esto, estableciendo la preeminencia del sujeto por sobre lo real;

[...] sería la negación absoluta de cualquier noción de un mundo objetivo que exista con independencia de nuestras tentativas de llegar a conocerlo, sustituyendo esta tradicional concepción objetivista por algún tipo de versión de una imagen bohriana, en la que el mundo está descrito por estados cuánticos, pero estos estados son de suyo relativos a la elección de una medición a realizar (Sklar, 1992: 323).

Esa mediación determinante del observador en la teoría cuántica hace que el cálculo probabilístico al que se recurre para poder explicar las relaciones de indeterminación de las

$6 \quad$ Popper destaca de Niels Bohr la introducción que realiza a la física atómica con el principio de complementariedad, el cual-afirma el filósofo vienés- equivale a "renunciar" al intento de interpretar la teoría atómica como una descripción de algo (en 1972: 134). 
partículas elementales en su impulso y posición, resulte, sostiene Popper, en una interpretación subjetivista de la probabilidad, que es producto de las propias consideraciones del observador, de su ignorancia o conocimiento imperfecto de las mencionadas relaciones subatómicas. A este respecto, el físico contemporáneo Eyvind Wichmann afirma:

Cuando aceptamos la mecánica cuántica como teoría nuestra, introducimos con ello una indeterminación en la manera como describimos la naturaleza [...] habrá siempre una medición el resultado de la cual no se puede predecir en ningún caso particular [...] si las mediciones se hicieran de una mejor manera, las conclusiones serían diferentes (Wichmann, 1986: 287).

Esto indicaría que la probabilidad de que suceda un acontecimiento -esto es, de que eventualmente el suceso se dé, y no solamente de que la probabilidad en su cálculo puramente formal resida en la ignorancia parcial del sujeto- dependería de la exigua evidencia que conforma el conocimiento del observador. Popper ejemplifica de la siguiente forma: "entonces $p(a, b)$ es, precisamente, el grado en el que nuestro conocimiento efectivo total $b$ justifica racionalmente un $a$ hipotético o dudoso" (2011a: 336). De acuerdo con esto, se hace patente una dependencia total en la información pertinente que el sujeto $b$ posea o no con anterioridad del suceso $a$, para así poder establecer el valor verdadero de $p(a, b)$, esto es, el valor para poder determinar la posibilidad real de $p(a, b)$. Esta probabilidad condicional subjetivista presenta varias inconsistencias lógico-matemáticas; como por ejemplo, que la probabilidad dependería condicionalmente del espectador, las circunstancias y la evidencia, dando como fórmula lo siguiente: $p$ $(a, e, c, e)$, lo cual no se ajusta a la fórmula propia del cálculo de 
la probabilidad condicional, a saber, $p(a, b)$. Pero la principal crítica de Popper se dirige contra la primacía-dependencia del conocimiento inductivo o a la ignorancia por parte del sujeto, lo que para el filósofo vienés es inamisible, y que la realidad de suyo no puede acaecer o constituirse ontológicamente por la arbitrariedad y limitación del sujeto cognoscente. A esto el filósofo Mario Bunge ratifica de la siguiente manera:

Si las probabilidades no son sino credibilidades (o verosimilitudes, o grados de certidumbre), ¿cómo es que todas las probabilidades que encontramos en la ciencia fáctica y en la técnica son probabilidades de estados de cosas concretas (átomos, campos, organismos, sociedades, etc.) o probabilidades de sucesos que ocurren en cosas concretas, independientemente de las credibilidades que el personalista pueda asignarles a las hipótesis acerca de dichos hechos? (Bunge, 1986: 136).

Por su parte, la teoría de la probabilidad objetiva frecuencial, en su labor interpretativa-explicativa de la teoría cuántica de los sucesos estocásticos-, tampoco es una opción viable para Popper, dado que ésta no cumple con sus exigencias realistas, sino que dicha opción objetiva de la probabilidad resulta en un instrumentalismo ensimismado que no llega a descubrir la realidad. Popper la define con estos términos: "La interpretación frecuencial siempre considera la probabilidad como relativa respecto a una sucesión que se supone «dada»: y opera con el supuesto de que una probabilidad es una propiedad de una sucesión dada" (2011a: 395). Es claro que esta interpretación probabilística es objetiva porque se basa ya no en el conocimiento que posea o no el sujeto, sino más bien en la observación de una sucesión de acontecimientos objetivos; y es precisamente en esa sucesión en donde reside 
su problema, por cuanto la interpretación frecuencial depende de muestreos de sucesiones infinitas o muy largas que a su vez tendrían que ser efectivas-observadas, situación ya de por sí imposible, y en este caso no se da una explicación del por qué de un suceso singular sino hasta dado todo el conjunto de los sucesos observados, lo cual estaría dejando por fuera el elemento ontológico que daría lugar o explicaría el por qué de una determinada frecuencia estadística. Aquí es donde surge la propuesta popperiana de las propensiones metafísicas, o lo que es lo mismo tendencias o disposiciones como las propiedades disposicionales objetivo-reales de un determinado suceso, que al mismo tiempo podrían ser contrastadas por medio de frecuencias y que también darían lugar a las mismas frecuencias estadísticas. "Al par que una frecuencia es la frecuencia de sucesos actuales de cierto tipo, una probabilidad puede medir la posibilidad de un hecho, o más bien la intensidad de semejante posibilidad" (Bunge, 1986: 139-140). En consecuencia, podemos inferir que la probabilidad como frecuencia se reduce a un mero muestreo o porcentaje acerca de una colección de sucesos que se suponen como efectivos, y que no hacen una verdadera descripción o descubrimiento del mundo, sino estrictamente una acción operativa sobre datos.

Lo propio del instrumentalismo, según Popper, consiste en ser una ayuda para poder deducir $b$ a partir de $a$; su función relevante es hacer posible las inferencias, los cálculos, las mediciones, etcétera, pero que en última instancia se reducen a sus mismas operaciones. El problema del instrumentalismo, siguiendo a Popper, radica en considerar la ciencia como un medio para formular deducciones y hacer predicciones sobre sucesos y nada más que eso; su foco central no está en la descripción-comprensión de la realidad, sino en la teoría mis- 
ma, en su coherencia interna, en su eficacia y su consiguiente aplicabilidad. Aquí reside una gran diferencia entre las teorías científicas "puras" y las reglas para el cálculo tecnológico (el puro instrumentalismo), porque estas últimas no pueden ser contrastadas para su debida refutación; no obstante, lo que sí sucede con ellas es que pueden no ser aplicables o fallar en algunos casos, pero de igual forma, podrían seguir siendo utilizadas en otros. De esta forma, a diferencia de las teorías científicas que buscan indagar en la realidad y realizar conjeturas verosímiles, las reglas instrumentalistas se convierten en un fin en sí mismo, sobrevalorando las operaciones que realizan y olvidándose precisamente de la realidad que las originó, de las conceptualizaciones realistas que conformaron las propias reglas; pero en eso mismo ha caído el instrumentalismo científico con su negación de la independencia, primacía y verdadera descripción de lo real por sobre una conciencia productora, y, en consecuencia ineludible a esto, se instaura la afirmación del antirrealismo. "Pero según el antirrealista, el proponente no afirma la teoría: la despliega y reclama ciertas virtudes para ella. Estas virtudes pueden no llegar a la verdad: adecuación empírica; quizás alcance, aceptabilidad para varios propósitos"' (Van Fraassen, 1996: 26).

Esta tendencia antirracionalista del instrumentalismo, como sentencia Popper, implica que no hay acceso por parte de la razón humana hacia la realidad, que no hay conceptualización ni comprensión ni progreso científico o, en último término, que no hay conocimiento verdadero, es decir, no hay verdad (verosimilitud) en la ciencia: sólo hay utilidad. Pero la filosofía de Popper nos recuerda que esto no coincide con la historia de la ciencia, la cual ha sido partícipe de alcances parciales de la verdad, pero con todo alcances significativos -esto a partir de un realismo metafísico asumido- que han contribuido 
al progreso humano, especialmente, ofreciendo una concepción verdadera del mundo.

La teoría de las propensiones

Es desde este contexto científico-filosófico que surge la teoría de las propensiones, como una interpretación de la probabilidad, para dar una explicación-completitud de la teoría fundamental de la física, que es la que ve por la estructura fundamental de la materia, y cuyo fin es dar una explicación coherente, racional y objetiva de los sucesos estocásticos propios del mundo subatómico, que fundamentalmente son sucesos singulares. Estos sucesos, en tanto que estados reales de partículas para su explicación causal ${ }^{7}$, requieren ser abordados desde una perspectiva ya no instrumentalista, sino metafísica. De ahí que la interpretación propensivista sea catalogada, según Popper, como un programa metafísico de investigación -no contrastable-, debido a que se entiende como una concepción general sobre la estructura del mundo, y, que de igual manera, busca determinar si se logra o no la precisión explicativa en la teoría científica.

Así pues, la teoría de las propensiones se presenta como el intento de superación de la teoría frecuencial de la probabilidad -la cual sigue siendo considerada por Popper como una teoría viable-, que no alcanza a dar cuenta de lo real-físi-

$7 \quad$ A pesar de que se sostiene que existen explicaciones no causales, como es el caso de las explicaciones estructurales, que apelan a leyes de coexistencia, también se afirma que dichas explicaciones dependen de explicaciones causales previas, las cuales están en conexión necesaria con tales explicaciones que no apelan a la causalidad. Igualmente, se alega que de no haber referencia a la causalidad en una explicación teórica, tal teoría sería altamente predictiva pero no explicativa. Para un desarrollo completo y actual de este tema véase (Diez y Moulines, 2008: 229-280). 
co, sino que se remite a ser una propiedad del conjunto, una valoración de una probabilidad actualizada. "Mientras que el punto de vista frecuencial toma la probabilidad para pertenecer colectivamente a la secuencia que constituye la referencia de clase o el colectivo, el enfoque propensivista toma la probabilidad para pertenecer distributivamente como sucesos individuales de esa clase" (Kyburg y Teng, 2003: 78). Para Popper, las propensiones cumplen la función de explicar o dar razón de la frecuencia relativa, del porqué de ciertas propiedades estadísticas; esto significa que las propensiones darían lugar, de acuerdo a unas condiciones específicas, a la posibilidad real -y su respectiva valoración posterior- del surgimiento de sucesos singulares aleatorios; esto nos llevaría a afirmar que el fundamento del comportamiento aleatorio de la realidad microfísica estaría en las propensiones. Es en este punto en el que nuestro filósofo basa toda su argumentación para proponer la teoría de las propensiones como la mejor interpretación de las probabilidades singulares -mismas que fueron interpretadas, afirma Popper, por los teóricos cuánticos como formalmente singulares, esto es, como enunciados estadísticos sobre colectivos-, esto debido a que la teoría cuántica trata con mediciones a entidades reales, elementales-singulares en su comportamiento inexacto ${ }^{8}$, dando lugar esto a la confirmación de la presencia del indeterminismo en la realidad física, además de la probabilidad objetiva de tipo singular. De manera que las propensiones se consti-

$8 \quad$ E.H. Wichmann afirma: “Una partícula física real es un ente irreducible único, y sus propiedades ondulatorias, al igual que sus propiedades corpusculares, son manifestaciones de aspectos diferentes de su naturaleza intrínseca". Además, concluye que en cuanto a las predicciones exactas de las mediciones descritas por la función de onda, "introducimos con ello una indeterminación en la manera como describimos la naturaleza" (ver Wichmann, 1984: 248, 287). 
tuyen como la disposición probabilista de la estructura física fundamental, y que igualmente, a partir de sucesos singulares, dan lugar a una teoría cuántica que describe verdaderamente la estructura primordial de lo real. Así, afirma Popper:

Pero las probabilidades que la teoría determina son siempre las propensiones de las partículas a adoptar cierto estado bajo ciertas condiciones. [...] Así pues, podemos describir el mundo físico como consistente en cambiantes propensiones al cambio. [...] Este enfoque contiene la sugerencia de una teoría de la materia o de las partículas, según la cual éstas se interpretan como realizaciones de potencialidades o propensiones (2011c: $145,176)$.

Por su parte, las propensiones son evaluadas básicamente con el cálculo de probabilidades condicionales, es decir, la probabilidad condicional de $a$ dado $b-p(a, b)-$, o en otros términos, la propensión o tendencia que posee una determinada cosa a sucederse del estado $b$ al estado $a$. Esto significa, afirma Popper, que toda disposición experimental genera propensiones, las que a su vez producen sucesiones posibles, las cuales son contrastadas por frecuencias estadísticas. De esta forma, cuando un suceso singular tiene una propensión a ocurrir efectivamente, esto es, $p(a, b)=1$-dadas las condiciones generadoras-, esa ocurrencia singular se interpreta desde sus mismas propiedades disposicionales o propensiones, y correspondientemente es medida por una frecuencia estadística potencial o virtual ${ }^{9}$. Se establece, así, que son las mis-

9 Popper sostiene que la frecuencia virtual se utiliza para conjeturar las mediciones de secuencias muy cortas o muy largas o que varían con el tiempo, las cuales se someten a prueba con secuencias reales de sucesos. 
mas propensiones las que ocasionan las sucesiones con frecuencias, manifestando con ello las probabilidades efectivas.

La estructura ontológica de las propensiones

A partir de las precedentes argumentaciones popperianas acerca de la interpretación de la probabilidad como propensión, podemos evidenciar que la teoría de las propensiones se presenta como una teoría metafísica, o en otros términos, como una ontología de la probabilidad.

Primeramente, las propensiones son realidades físicas que ocasionan ciertos fenómenos, son tendencias o disposiciones de situaciones físicas que se dan efectivamente en la realidad. Su constitución, en su referencia a lo real, es conjuntiva, es decir, que sus propiedades son relacionales, que involucran, afirma Popper, "al menos dos cosas"10 (2011a: 399), las cuales remiten hacia un sistema físico compuesto en el que las condiciones específicas de una situación física provocan determinada singularidad aleatoria. A esto, Popper precisa que las propensiones no son propiedades inherentes ni exclusivas a una cosa concreta, como por ejemplo un dado o una moneda, sino que éstas son algo más abstracto, de carácter primigenio, a manera de unas propiedades ocultas análogas a las fuerzas newtonianas, que se extienden a toda la realidad física elemental, esto como su componente productor y configurador, o más precisamente, que "la estructura de lo real son campos de propensiones"' (Queraltó, 2004: 269); además, esto las constituye, según nuestro filósofo, en

10 Craig Dilworth subraya que una implicación importante de la interpretación propensivista es que las probabilidades son propiedades objetivas de un estado de cosas y no dependientes del conocimiento de un observador (ver Dilworth, 2006: 175). 
una hipótesis metafísica ya no contrastable. Así, afirma Popper acerca de las propensiones:

Propongo interpretar la probabilidad objetiva de un suceso singular como una medida de una propensión objetiva, de la fuerza de la tendencia, inherente a la situación física especificada, a realizar el suceso, a hacer que ocurra. [...] son propiedades relacionales de la situación objetiva total; propiedades ocultas de una situación cuya dependencia precisa de la situación sólo podemos conjeturar (2011a: 434, 398).

Con esto, el formalismo matemático, propio de las interpretaciones instrumentalistas de la teoría cuántica, ha dado paso a una especulación situada en el ámbito propiamente metafísico. Estas propensiones metafísicas, como el mismo filósofo vienés las llama, pertenecen a un programa metafísico de investigación, porque, en palabras de Popper: "son el resultado de una conceptualización general sobre la estructura del mundo $\mathrm{y}$, al mismo tiempo, de concepciones generales sobre la situación de los problemas de la cosmología física" (2011c: 177); problemas que pueden ser solucionados por ideas metafísicas, satisfactorias o aceptables, dando con ello las respuestas que proporcionen el perfeccionamiento y avance a los problemas planteados por la teoría física. Con esto, el filósofo busca lograr una integración entre ciencia y filosofía, necesaria para alcanzar esa perspectiva coherente del mundo físico.

Es así como la interpretación propensivista, con su marcado carácter ontológico, puede solucionar los problemas o, en términos popperianos, "el gran embrollo cuántico", introducido por la teoría cuántica en su disociación con el realismo y el objetivismo. Así pues, la interpretación propensivista intenta dar la completitud al formalismo matemático, 
el cual no puede acceder a la realidad y, por consiguiente, presenta una imagen imprecisa de la misma. Popper critica el formalismo experimental y dependiente del observador, sostenido principalmente por Niels Bohr, el cual defiende la dualidad onda-partícula, necesaria según el físico danés, para dar una descripción exhaustiva de los fenómenos, esto a pesar de que cada una de las propiedades se manifiesta en situaciones diferentes y excluyentes ${ }^{11}$. Para Popper, esta tesis presenta algunas inconsistencias de tipo lógica, epistemológica y principalmente ontológica, y que dan lugar a un dualismo en la materia (infringiendo con ello el principio de identidad), a la dependencia del observador y a la inaccesibilidad a la verdadera estructura de lo real. A partir de esto, la propuesta popperiana busca explicar el comportamiento cuántico de las partículas con su ambigüedad dual postulada, superando el formalismo estadístico, esto con las propensiones reales que son las que producen el dinamismo de las partículas, y que a su vez son propensas a adquirir un cierto estado bajo ciertas condiciones, y también las ondas, que en este caso y, según el filósofo, "describen propiedades disposicionales de las partículas” (2011c: 145). “La realidad, finalmente, estará compuesta de partículas y campos de propensiones, los cuales son objetivos y medibles precisamente a través de las leyes estadísticas" (Queraltó, 1996: 124). Aquí, el filósofo vienés hace una osada especulación puramente filosófica aplicada a

11 Niels Bohr, haciendo referencia al Principio de Complementariedad, afirma: "sirve para simbolizar la limitación fundamental con que se tropieza en física atómica de la existencia objetiva de los fenómenos independiente de los medios de observación. [...] Los conceptos a los que se hace referencia son los de onda y de partícula que, a pesar de que "representan" manifestaciones diferentes y contrarias son necesarios para una descripción exhaustiva de los fenómenos, ambos explican características igualmente importantes de los fenómenos luminosos"' (ver Bohr, 1964: 10, 7). 
la teoría física para concluir que las propensiones producen el carácter tendencial -no dual- o variable del estado de las partículas en su situación estructural de conjunto. Popper lo expone con estos términos:

La concepción de que las propensiones son reales; que son descritas por ecuaciones de campos; que las partículas pueden ser producidas por propensiones y que al menos hasta cierto punto las partículas son propensiones (2011c: 209).

\section{Causalidad indeterminista}

Esta propuesta especulativa como respuesta al problema ontológico de la teoría cuántica nos permite trazar unas líneas argumentativas que nos llevan a poder identificar en las propensiones una causalidad de tipo indeterminista. Para ello, señalaremos los elementos clave por los que creemos que es posible constatar una causalidad indeterminista en la teoría de las propensiones popperianas.

En primer lugar, señalamos que ciertamente Popper no utiliza en sus escritos una nomenclatura concerniente a la causalidad, en este caso para referirse específicamente a las propensiones, y esto creemos que es debido, primeramente, al rechazo del filósofo vienés hacia el determinismo metafísico, mismo que ha sido comúnmente asociado a la causalidad; como el mismo Popper afirma: "Otra forma en la que el sentido común aborda la idea del determinismo es por medio de la idea popular de causalidad" (2011b: 32); y es por ello que evita utilizar el concepto causalidad, para así escaparse de asociaciones con esta postura metafísica. De igual forma, su oposición al esencialismo también influye en su actitud resistiva al uso de la terminología, debido a la pretensión 
esencialista de dar "explicaciones últimas" -aquellas que no dan lugar a planteamientos ulteriores-, y de responder a las preguntas de tipo “¿qué es?" -las que pretenden llegar hasta la esencia o verdadera naturaleza de las cosas-, lo cual no es aceptable para el filósofo. Por otra parte, Popper deja claro desde La lógica de la investigación científica que el concepto "principio de causalidad", por ser un concepto metafísico, queda fuera del ámbito de la ciencia y, por ello mismo, el pensador austriaco queda influido para no adoptar su uso ya desde sus primeros escritos de carácter estrictamente epistemológico ${ }^{12}$. Pero esto no implica el rechazo a tal principio, ya que éste, según el filósofo, es imprescindible para el científico en su intento por encontrar leyes que le permitan deducir explicaciones y predicciones; e incluso, el mismo Popper sostiene que no existe incompatibilidad en el enlace entre leyes causales o deterministas (enunciados precisos) y leyes probabilísticas (predicciones frecuenciales). Así, afirma:

No existe nada en estas dos tareas [leyes deterministas y leyes probabilísticas] que las haga mutuamente incompatibles en ningún respecto: sin duda alguna no ocurre siempre que presentemos enunciados precisos no debemos hacer hipótesis frecuenciales, pues a algunos de aquéllos corresponden macro-leyes deductibles de asunciones frecuenciales (2013: 290).

Y continúa:

[...] ni cesaremos en nuestros intentos de explicar causalmente todo tipo de acontecimientos que podamos describir: esta regla

12 Véase el apartado 12 Causalidad, explicación y deducción de predicciones, en Popper, 2013: 72-74. 
guía al investigador científico en su tarea. [...] pero negar la causalidad sería lo mismo que intentar persuadir al teórico de que abandone su búsqueda (2013: 73-74, 291).

De esta manera, vemos que Popper no rechaza el principio de causalidad, entendido éste como las condiciones iniciales que permiten explicar un suceso específico (efecto), el cual puede ser probabilístico; y de esta forma reconocemos un primer vínculo entre causalidad e indeterminismo. En vista de ello, la explicación como el objetivo de la ciencia debe dar razón de cualquier cosa que requiera explicación, esto a partir de dichas condiciones y leyes generales (explicans), para así poder deducir el enunciado que explique y prediga un acontecimiento concreto (explicandum). Así pues, explicación y causalidad están vinculadas entre sí como el medio que le permite a la ciencia avanzar en el conocimiento y comprensión de la realidad; asimismo, la propia ciencia requiere ideas y conceptos metafísicos para que pueda adquirir un sentido coherente y completo en su descripción teórica del mundo físico. Todo esto nos permite avanzar hacia la propuesta de la concepción causal-indeterminista.

Hemos visto que a partir de las características asociadas a las propensiones, en su función interpretativa y explicativa, se manifiestan ciertas propiedades que corresponden debidamente a la causalidad, como por ejemplo que las propensiones se constituyen como la causa de fenómenos, propiedades, comportamientos, ocurrencias, etcétera, de los sucesos singulares en su situación de conjunto. Es la propensión la que influye realmente -ontológicamente- en el realizarse, en el sucederse de los fenómenos, en este caso probabilísticoindeterminados. De esta forma, la producción -característica principal de la causalidad- de un efecto físico dependería de 
las propensiones, constituidas, así como las condiciones iniciales que explicarían el suceder dicho efecto; en palabras de Popper: "que las partículas pueden ser producidas por propensiones" (2011c: 209). Vemos, incluso, ya en uno de sus últimos escritos, cómo el filósofo explícitamente postula una clara relación entre causa y propensión, en un sentido de condición límite de propensión; así, asegura: “La causación no es sino un caso especial de propensión: aquél en el que la propensión es 1, una fuerza o demanda determinante de realización" (1992: 43); por supuesto, dicha realización o suceso no puede ser predicho ni conocido sino hasta que acontece efectivamente.

Por su parte, la causalidad, en tanto condiciones iniciales, no está, según nuestro filósofo, asociada necesariamente al determinismo, dado que para predecir o explicar un suceso cualquiera con precisión, el determinismo científico requiere que las condiciones iniciales sean suficientemente precisas, situación, que como es bien sabida, con la teoría cuántica no se da. En consecuencia, las condiciones iniciales nunca se conocen con precisión absoluta, y esto da como resultado la imprecisión en la predicción, a saber, el indeterminismo. A pesar de esto, las condiciones iniciales o la causalidad siempre prevalecen, pero no así el determinismo, esto a causa de que la permanencia de las condiciones iniciales es indispensable para hacer inteligibles los fenómenos físicos que se manifiestan probabilísticamente $\mathrm{y}$, que al mismo tiempo, formulan preguntas de tipo estadístico, lo que requiere, por tanto, una contestación desde una teoría probabilista. Todo esto demanda una causalidad de tipo indeterminista debido a la producción fluctuante de los resultados. A este respecto, Lawrence Sklar afirma: “Cuando explicamos probabilísticamente un suceso es ubicar dicho suceso en una estructura de relaciones causales, donde la estructura revelada es probabilística, bien porque las rela- 
ciones causales son intrínsecamente indeterministas" (Sklar, 1994: 158). Bien puede afirmarse que la causalidad indeterminista debe provocar diferentes efectos que pertenecen a alguna clase específica de posibles resultados ${ }^{13}$. De aquí que las propensiones adoptan y propician una serie posible de estados aleatorios con sus correspondientes valores, dadas determinadas condiciones y, uniendo así, el indeterminismo o la imprecisión de los sucesos, con la regularidad causal, que es la que produce y distribuye las probabilidades. Así lo confirma Popper:

[...] el determinista se ve forzado a recurrir a una hipótesis incontrastable sobre fluctuaciones ocultas de las condiciones iniciales, una explicación en términos de propensiones, cuya presencia pueda contrastarse estadísticamente, puede resultar preferible. [...] en general, consideramos que las propensiones asumen, bajo determinadas condiciones, uno u otro de una serie de estados «posibles» (o «virtuales») (2011b: 116-117).

Son esas condiciones iniciales ocultas, las propensiones, las que van a poder explicar teóricamente y a fundamentar ontológicamente los diferentes fenómenos estocásticos, e igualmente a poder distribuirlos de distintas maneras dentro de una probabilidad específica. En conclusión, Popper declara que: "suponemos que es válida una teoría de medida no estadística de la probabilidad para la distribución de nuestras condiciones iniciales y que esta teoría de la probabilidad tiene que interpretarse físicamente (yo sugiero que por propensiones)"14 (2011b: 121).

13 Ideas con relación a este tema se encuentran desarrolladas en Fetzer (1988: 109 y ss).

14 En continuidad con la propuesta popperiana, el filósofo de la ciencia Wesley Salmon argumenta que la propensión como causalidad probabilista sería la 
El universo abierto

Confirmado el carácter indeterminista de la realidad física con la teoría cuántica e, igualmente, esta última asimilada a un realismo epistemológico y metafísico y, seguidamente completada y depurada de discontinuidades causales y dualismos ontológicos, quedan constituidas las propensiones como la base metafísica de todo ese ensamblaje filosófico. Con ello se ha alcanzado una visión general de la realidad física en su estructura elemental, a saber, un universo indeterminista pero ordenado causalmente por propensiones $\mathrm{y}$, consecuentemente, un universo abierto a nuevas posibilidades creativas y con regularidad dinámica; en términos popperianos: “El indeterminismo y la interpretación propensivista de la probabilidad nos permiten trazar una nueva imagen del mundo físico" (2011c: 175). "Y esto se ha llevado a cabo haciéndolo compatible [al indeterminismo] con la causalidad general, lo cual era especialmente importante" (Queraltó, 1996: 164). Es decir, que con las propensiones físicas como fundamento causal se sientan las bases que hacen posible la construcción de una integrada imagen cosmológica.

Esa imagen resultante es la de un universo abierto en el que no hay una predictibilidad absoluta, lo que significa que no todos los sucesos están absolutamente predeterminados o no hay un conocimiento exhaustivo de las condiciones ini-

responsable de provocar el comportamiento dinámico de las partículas elementales, provocando así una distribución probabilística de dicha dinámica, esto en todas las posibles direcciones con el fin de interactuar con otros procesos causales y, con ello, dando lugar a una determinación de probabilidades de varios resultados cuando las interacciones causales ocurren. En definitiva, Salmon define la propensión con estos términos: "una propensión es a la vez una tendencia causal y probabilística", y seguidamente, agrega que hay dos tipos de acción causal, éstas son: producción y propagación (ver Salmon, 1998: 204ss). 
ciales, es por ello que el conocimiento científico no puede dar razón de lo que ha de suceder, como si la ocurrencia de los sucesos tuviera que darse irrevocablemente, porque ya están predeterminados en un pasado ahora inmutable. Con esto, el filósofo de Viena confirma que no hay simetría entre pasado y futuro, éste no depende de lo que sucedió en aquel, "el futuro -dice Popper- no está todavía completamente fijado; al contrario del pasado, que está cerrado, por decirlo así, el futuro está todavía abierto a influencias; todavía no está completamente determinado" (2011b: 79-80). De manera que siempre habrá lugar para nuevas posibilidades de realización, nuevas realidades, para cambios en relación con un pasado cerrado, e incluso al presente. Las futuras faces del universo son independientes de los fenómenos físicos ya acontecidos, los cuales -recordamos- son producto de las propensiones. Así, se pronuncia Popper a este respecto:

Las propensiones futuras están determinadas en cada instante, pero sólo las propensiones. (Ésta es la razón de que sean aplicables las relaciones de dispersión). Al irse realizando esas propensiones, en la forma de partículas con posiciones y momentos, las posibilidades abiertas se cierran. Así, la posibilidad de cálculo de las realizaciones pasadas y presentes, difiere de la del futuro (2011c: 216).

Este futuro abierto se corresponde con el cambio constante que permea en el universo, cambio que también se apoya (se explica) en las propensiones, ya que son ellas las que actualizan las diferentes posibilidades o potencialidades del mundo físico, para que puedan alcanzar el estado de realización y, evidentemente, de una manera distributivo-probabilista; Popper lo dice así: "podemos describir el mundo físico como consistente 
en cambiantes propensiones al cambio" (2011c: 216). Este devenir constante del universo es el que lo conduce hacia su evolución $\mathrm{y}$, desde ella, a un resultante universo emergente posibilitador de nuevas producciones, el cual explicaría la gran diversidad de entidades, organismos, elementos, la misma singularidad humana y sus producciones, y demás realidades que le conforman. En razón de esto, Popper reconoce que:

He ofrecido aquí una visión del mundo distinta (según la cual el mundo físico es un sistema abierto) compatible con la consideración de la evolución de la vida como un proceso de ensayo y supresión de errores. Además, nos permite comprender racionalmente, aunque no plenamente, la emergencia de novedades biológicas, así como el desarrollo del conocimiento y la libertad humanos (2010: 302).

\section{Los Tres Mundos del universo abierto}

A esta altura, concluimos haciendo mención del planteamiento de los Tres Mundos como resultado de la descripción real del mundo físico por parte de la ciencia y de la mano de la filosofía. El universo abierto, emergente y en devenir, ha dado lugar a una realidad contenida de elementos diversos e interrelacionados entre sí o, más exactamente, con un mutua acción causal. Lo que Popper se propone es explicar dicha variedad de productos surgidos del indeterminismo y las propensiones. Para ello, diferencia la gran pluralidad ontológica del universo abierto de la siguiente manera:

[...] el mundo consta al menos de tres submundos ontológicamente distintos: el primero es el mundo físico o de los estados físicos; el segundo es el mundo mental o de los estados mentales; 
el tercero es el de los inteligibles o de las ideas en sentido objetivo, el mundo de los objetos de pensamiento posibles (2010: 189).

Aquí, el filósofo plantea que el universo abierto está constituido por una emergencia de productos, que a su vez componen tres niveles distintos (Mundos 1, 2, 3), pero interrelacionados entre sí, esto a partir del basamento ontológico de las propensiones, que -como hemos dicho-, son las que posibilitan una compatibilidad entre el indeterminismo y la causalidad, además de ser el sustento de esta emergencia e interacción. Popper sostiene que la dimensión emergente de la realidad ha dado lugar al surgimiento procesual y ascendente de Mundos diferentes -de ahí los términos 1, 2, 3-; es decir, que primeramente surge el Mundo físico, luego el Mundo de los estados de la conciencia y, por último, el Mundo de los productos del pensamiento. Con esto, se puede apreciar una especie de direccionalidad teleológica del universo abierto que, a su vez, se sustenta en una interrelación causal, o lo que es lo mismo, que la dinámica progresiva presente en los Tres Mundos es lograda por la apertura causal que los constituye. Así, queda confirmado:

Lo que realmente necesitamos es la tesis de que el «Mundo 1» es incompleto; que puede ser influido por el «Mundo 2»; que puede interactuar con el «Mundo 2», o que está causalmente abierto hacia el «Mundo 2», y de ahí también hacia el «Mundo 3» (2011b: 148-149).

Con todos estos constructos especulativos del filósofo austriaco, vemos que la configuración de una concepción general y equilibrada de la realidad, o bien, de una cosmología consistente, requiere obligadamente de la correlación entre ciencia y filosofía, entre el conocimiento físico y el metafísico, 
$\mathrm{y}$, por ello, las teorías o conjeturas que buscan describir y explicar la realidad y solucionar sus problemas fundamentales no lo deben apelar a reduccionismos ni dogmatismos limitados. Del mismo modo, su teoría propensivista tiene la viabilidad de asumir la condición de fundamentación ontológica de la realidad física, adjudicándose filosóficamente el poder dar razón de un universo abierto -indeterminista- y diverso, pero con orden y regularidad ontológica. Finalmente, con la interpretación propensivista, Popper ofrece una respuesta al problema de la incompletitud instrumentalista, proporcionando filosóficamente lo que la teoría científica demanda para una resolución acorde con la aproximación a la verdad.

Bibliografía

Bohr, N. (1964). Física atómica y conocimiento humano. Madrid: Aguilar.

Bunge, M. (1985). Racionalismo y realismo. Madrid: Alianza Editorial.

Diez, J.A. y Moulines, C.U. (2008). Fundamentos de filosofía de la ciencia. Barcelona: Ariel.

Dilworth, C. (2006). The Metaphysics of Science, An Account of Modern Science in terms of Principles, Laws and Theories. Dordrecht: Springer.

Fetzer, J.H. (ed.). (1988). Probability and Causality, Essays in Honor of Wesley C. Salmon. D. Reidel Publishing Company, Dordrecht, Holland.

González, J.W. (2004). Karl R. Popper: Revisión de su legado. Madrid: Unión Editorial.

Kyburg, H.E. Jr. y Teng, C.M. (2003). Uncertain Inference. Cambridge: Cambridge University Press. 
Hempel, C.G. (2005). La explicación científica. Estudios sobre la filosofía de la ciencia. Barcelona: Paidós.

Martínez Solano, J.F. (2005). El problema de la verdad en K.R. Popper: reconstrucción histórico-sistémica. La Coruña: Netbiblo.

Popper, K.R. (2013). Lógica de la investigación científica. Madrid: Tecnos.

Popper, K.R. (2011a). Realismo y el objeto de la ciencia, Post Scriptum a la lógica de la investigación científica. Vol. I. Madrid: Tecnos.

Popper, K.R. (2011b). El universo abierto. Un argumento a favor del indeterminismo, Post Scriptum a la lógica de la investigación científica. Vol. II. Madrid: Tecnos.

Popper, K.R. (2011c). Teoría cuántica y el cisma en Física, Post Scriptum a la lógica de la investigación científica. Vol. III. Madrid: Tecnos.

Popper, K.R. (2011d). Búsqueda sin término. Una autobiografía intelectual. Madrid: Tecnos.

Popper, K.R. (2010). Conocimiento objetivo. Un enfoque evolucionista. Madrid: Tecnos.

Popper, K.R. (1992). Un mundo de propensiones. Madrid: Tecnos.

Popper, K.R. (1972). Conjeturas y refutaciones. Barcelona: Ediciones Paidós.

Queraltó, R. (2004). Realismo epistemológico y teoría de las propensiones en Popper. En Ciencia, sociedad y mundo abierto, Homenaje a Karl R. Popper (247-278). En Eugenio Moya (Ed.). Granada: Editorial Comares.

Queraltó, R. (1996). Karl Popper, de la epistemología a la metafísica. Universidad de Sevilla: Secretariado de publicaciones.

Reguera, I. (1995). El tercer mundo popperiano. Extremadura: Universidad de Extremadura. 
Salmon, W.C. (1998). Causality and explanation. Nueva York: Oxford University Press.

Sklar, L. (1994). Filosofía de la física. Madrid: Alianza Editorial. Sklar, L. (1993). Physics and Chance, Philosophical issues in the foundations of statistical mechanics. Cambridge: Cambridge University Press.

Sklar, L. (1992). Filosofía de la física. Madrid: Alianza Editorial. Van Fraassen, B.C. (1996). La imagen científica. México: Editorial Paidós Mexicana.

Wichmann, E.H. (1986). Física Cuántica. Barcelona: Editorial Reverté. 


\section{Resumen}

La concepción global de la realidad es una labor muy ambiciosa, pero a la vez necesaria y realizable, mas nunca definitiva y, claro está, desde la integración de saberes. El proyecto filosófico de Karl Popper busca construir una cosmología que posibilite una imagen unificada de la realidad, y que a la vez explique la gran diversidad de elementos que la componen. Para Popper esta labor es sólo posible desde una interacción entre ciencia y filosofía -en este caso de la metafísica-, para así lograr esa visión de conjunto, describir la realidad y avanzar en su conocimiento. El filósofo vienés busca dar completitud a la teoría científica fundamental de la realidad física, y para ello propone un programa metafísico de investigación, las propensiones y, a partir de ellas, la posibilidad de lograr su cometido.

Palabras clave: teoría científica, probabilidad, indeterminismo, causalidad, propensiones, emergentismo.

\section{Abstract}

The global conception of the reality is a very ambitious, require and achieve task, most never ending, and of course, from the integration of knowledge. The Karl Popper's philosophical project seeks to build a cosmology that enables a unified picture of reality and at the same time explain the great diversity of elements that it's composed. For Popper, this work is only possible from an interaction between science and philosophy -in this case of metaphysics- in order to achieve an overview, describing reality and go forward in their knowledge. The Viennese philosopher is searching to consummate the fundamental scientific theory of physical re- 
ality, for this he proposes a metaphysical research program, the propensities, and, from them achieving its mission.

Keywords: scientific theory, probability, indeterminism, causality, propensities, emergentism. 